\title{
Segmentation of Faces in Video Footage Using Controlled Weights on HSV Color
}

\author{
Osamu Ikeda \\ Faculty of Engineering, Takushoku University \\ 815-1 Tate, Hachioji, Tokyo, 193-0985 Japan \\ oikeda@cc.takushoku-u.ac.jp
}

\begin{abstract}
Accurate and reliable automatic segmentation of faces in video footages is often hard to succeed, leading instead to laborious and tedious interactive manual segmentation. This paper presents a segmentation method that uses a few controlled sets of the weights on HSV components. First, it is shown that HSV has advantages over RGB or YCbCr when segmenting a face in image in such that a binary pattern reflects as many features of the face as possible. Then, a face detection system is constructed, in which each time a significant scene change is detected segmentation is carried out for the beginning frame of a new scene using a few sets of the weights on HSV components, and resulting patterns are correlated with a typical face pattern. Computer experiments show that the successful detection rate is more than 95 out of 100 faces.
\end{abstract}

\section{Introduction}

Research on face detection has extensively been made in recent years especially in the fields of image processing and computer vision [1]. Rowley et al. [2] and Feraud et al. [3] used neural network-based methods, Schneiderman et al. developed a Naïve Bayes classifier [4], Osuna et al. proposed a method to train Support Vector Machines [5], and Turk et al. used eigenfaces [6]. Those algorithms are aimed at detecting or recognizing the face in image. The detection is required to be in real time in computer vision [7].

In the field of multimedia, on the other hand, the focus has been not just on their detection but also on identification of faces, people, or some specific objects in video images. Satoh et al., for example, tried to retrieve the name from the face or the face from the name in the video footages [8]. Since segmentation accuracy may affect the identification and images may be available in a limited duration of time, several improvements on segmentation have been reported, which combines temporal segmentation or tracking with spatial segmentation [9] or manual segmentation [10]. Long et al., for example, present a method that uses three consecutive frames to take into account motion, and they also adopt user interaction to cope with the situation where automatic detection fails [11].

In this paper, we present an improved segmentation method using HSV color and a construction of face detecting system. First, it is shown that HSV has advantages over $\mathrm{RGB}$ or $\mathrm{YCbCr}$ in segmentation capability and easiness in controlling the weights on the color. Then, a face detecting system is constructed based on the method, which detects a face in the video footage each time a significant scene change is detected. Computer experiments show that the successful detection rate is more than 95 percent. We also demonstrate its application to image retrieval from video footage. 


\section{Color Segmentation}

We require the segmented image to include as many features of the face as possible when comparing the segmentation capability for three colors, RGB, HSV, and $\mathrm{YCbCr}$, aiming at the construction of an image retrieval system. The comparison is made experimentally including the easiness to control the weights on the color components.

The color segmentation is meant to extract the image region that is continuous in the sense of eight neighborhoods with the sampled point within a given error as:

$$
\begin{aligned}
& \frac{w_{r}\left|r-r_{s}\right|+w_{g}\left|g-g_{s}\right|+w_{b}\left|b-b_{s}\right|}{w_{r}+w_{g}+w_{b}}<e_{R G B} \text { for RGB } \\
& \frac{w_{h}\left|h-h_{s}\right|+w_{s}\left|s-s_{s}\right|+w_{v}\left|v-v_{s}\right|}{w_{h}+w_{s}+w_{v}}<e_{H S V} \text { for } \mathrm{HSV} \\
& \frac{w_{y}\left|y-y_{s}\right|+w_{c_{b}}\left|c_{b}-c_{b_{s}}\right|+w_{c_{r}}\left|c_{r}-c_{r_{s}}\right|}{w_{y}+w_{c_{b}}+w_{c_{r}}}<e_{Y C_{b} C_{r}} \text { for } \mathrm{YCbCr}
\end{aligned}
$$

where the suffix s denotes the sampled point, each color value is normalized so that it varies between 0 and unity, and $w$ 's are weights. Face images in the video footages may have been illuminated with a variety of light sources and color adjustments, and background objects close to a face may often have similar colors to that of the face. In addition, face images may vary in skin color and texture, such as wrinkles, apart from frontal direction and size. So there may exist appropriate weights for each face image and they may have to be given in a controlled way.

The image region which satisfies Eq. (1) but is not continuous with the sampled point is included in the segmented image, if even a slightest part of the region is longitudinally sandwiched by the segmented part within a certain distance, as shown in Fig. 1. This extension may make the segmented image more informative, possibly, enough to recognize the person.

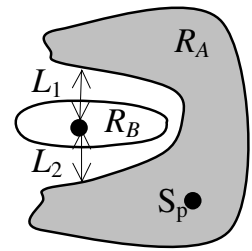

(a)

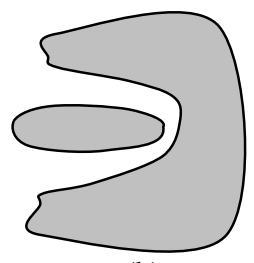

(b)

Fig. 1 The region $R_{B}$ in which Eq. (1) is satisfied is not continuous with the sampled point $\mathrm{S}_{\mathrm{p}}$. However, if there exists any pixel in $R_{B}$ whose distances $L_{1}$ and $L_{2}$ to $R_{A}$ are within a certain value, $R_{B}$ is added up to $R_{A}$.

\section{Face Detection System and Its Application to Image Retrieval}

A face detection system is constructed based on the segmentation method as follows:

(a) Scene change is detected in a video footage

(b) A sampling point is scanned on the beginning frame of a new scene

(c) At each sampling point segmentation is carried out for each set of the weights on the HSV components and for each segmentation error

(d) Each segmented image is made binary whose pattern is checked whether it satisfies the requirements as face and then it is correlated with a typical face pattern 
(e) The segmented face with the largest correlation value is output as the face for the frame

The scene change is detected by evaluating the difference between the neighboring frames as:

$$
D_{\text {scene }}(n)=\frac{1}{3 X_{I} Y_{I}} \sum_{x=1}^{X_{I}} \sum_{y=1}^{Y_{I}} \sum_{c=r, g, b}|i(x, y, c ; n)-i(x, y, c ; n-\delta n)|
$$

where $n$ is the frame number, $i$ is the image, $c$ is $r, g$ or $b$ component, and $\delta n(\geq 1)$ is the transition number of frames from one scene to another. The scene is judged to have changed if $D_{\text {scene }}>D_{t h}$ holds.

With a hope to detect faces with size $8 \times 12$ pixels or larger on a reduced frame of $76 \times 58$ pixels, we sampled each frame at 132 points within an appropriate region. The effects of the weights on the three HSV color components are examined in advance by manual segmentation, as will be shown. Based on the results, we use basically three sets of the weights to cope with a variety of face images. We also use several segmentation errors, especially, to cope with various lighting conditions.

The dimensions of the segmented image, $X$ and $Y$, are required to satisfy

$$
Y<2.5 X \text { and } X<1.25 Y
$$

Then the image is made binary and the resulting pattern is required to satisfy

$$
N>N_{e} \text { for eyes, } \quad N>N_{m} \text { for mouth }
$$

where $N$ is the number of vacancies in the regions assigned for eye or mouth in Fig. 2, and $N_{e}$ and $N_{m}$ may be proportional to the area of the segmented image:

$$
N_{e}=c_{e} X Y \text { for eyes, } \quad N_{m}=c_{m} X Y \text { for mouth }
$$

where $c_{e}$ and $c_{m}$ are constants. Then, the correlation is taken between the binary pattern, $P_{s}$, and a typical face one, $P_{k}$,

$$
C_{p}=P_{s} \otimes P_{k}
$$

where the former is fitted in size to the latter. In the case of $Y>2 X$, where a neck part may often be included in the segmented image, however, the fitting ratio in $y$ is made the same as in $x$, and the top part of the segmented face pattern is used for the correlation. The elements of the two patterns have values of +1 and -1 , so that $C_{p}$ takes a value in the range $[-1,1]$. The operation in Eq. (6) is repeated for the maximum number of candidates of \{number of segmentation errors $\} \times$ \{number of sets of the weights $\}$ x \{number of sampling points\}, among which the segmented image of the highest correlation value is output as the face for the frame.

We may often encounter situations where we want to retrieve scenes or images from an image at hand. This becomes possible if we modify the face detection system. First, a face part is segmented from the image at hand, and it is made binary with mean color values. Then, in the face detection process the color at each sampled image point, $\left(h_{s}, s_{s}, v_{s}\right)$, also is checked whether it is close enough to that of the key image, $\left(h_{k}, s_{k}, v_{k}\right)$, as

$$
\left|h_{k}-h_{s}\right|<e_{h},\left|s_{k}-s_{s}\right|<e_{s},\left|v_{k}-v_{s}\right|<e_{v}
$$




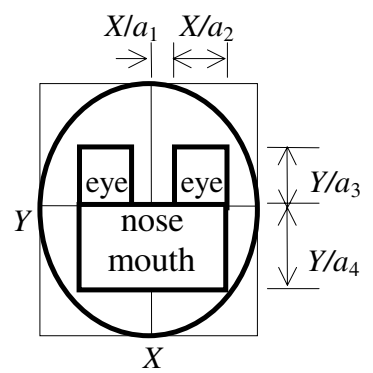

Fig. 2 The binary face pattern is required to have certain numbers of vacancies in the regions where eyes and mouth are supposed to be.

If the conditions are met, the sampling point is accepted and the segmentation is carried out. If not, the proximity in color is checked for the next sampling point. For each segmented image, average color values, $\left(r_{s}, g_{s}, b_{s}\right)$, are calculated and correlated with those of the input image, $\left(r_{k}, g_{k}, b_{k}\right)$, as

$$
C_{c}=\exp \left[-\frac{\left(r_{s}-r_{k}\right)^{2}+\left(g_{s}-g_{k}\right)^{2}+\left(b_{s}-b_{k}\right)^{2}}{6 \sigma^{2}}\right]
$$

The segmented image for the frame is selected based on the product of the correlation in color and that in pattern instead of the correlation in pattern alone.

$$
C=C_{c} C_{p}
$$

\section{Experiments}

First, the segmentation was manually carried out to compare the segmentation capability among RGB, HSV and $\mathrm{YCbCr}$ colors using several images, where the weights on the three color components are adjusted so as to include the faces' features as many as possible. A few typical results obtained are shown in Fig. 3. For the face in Fig. 3(a) all the three colors can segment the face well to a similar degree. It appears that the pattern obtained with RGB reflects her features slightly more than the other two. For the face in (b) HSV can separate the face from the surroundings, but neither RGB nor YCbCr can do that due to the closeness in color. In addition HSV appears to extract more of his face's features than the other two, and RGB does not distinguish the face from the hair. For the face in (c), RGB cannot separate the face from the surroundings due to the color similarity and gives a huge vacancy to his head. The two patterns by $\mathrm{HSV}$ and $\mathrm{YCbCr}$ are similar, but the pattern by HSV appears to more reflect his face's features than YCbCr. For the face in (d), RGB cannot distinguish her face from her hair due to the closeness in color, $\mathrm{YCbCr}$ has the same disadvantage as RGB and misses part of her face. On the other hand, HSV segments her face well regardless of the fact that her face color is very close to her hair color. Hence it is seen that HSV on average can segment faces more successfully and informatively than RGB or YCbCr. In addition, the three HSV components are independent parameters having clear meanings, so that HSV may be the easiest to control.

In the experiments of face detection, we edited video footages from various news programs to create one that consists of 100 different scenes having just one frame each, as shown in Fig. 4. We used six segmentation errors, 7, 9, 11, 13, 15 and 17 percent. A larger shading change on the face tends to need a larger error to segment, 
and a larger weight on $V$ also tends to need a larger error.

When a single set of the weights on the three HSV components was used, the successful detection rates were $84,90,95,95,81$ percent, respectively, for the weights $(1,1,5),(1,1,2),(1,1,1),(2,2,1),(5,5,1)$, where the pattern in Fig. 5(a) was used as typical face pattern. When we used either of the two sets of the weights, $(1,1,5)$, $(1,1,1)$ and $(5,5,1)$, and $(1,1,5),(1,1,1)$ and $(2,2,1)$, the rate increased to 97 . The segmented face images obtained for the second set are shown in Fig. 6, including the failed ones. The rate remained the same even if we use a different typical pattern in Fig. 5(b). We notice in Fig. 6 that the 14th or 60th segmented face is cut off due to the restricted region around the sampled point, which is allowed for the segmented image to exist, that part of the background image is included in the 30th, and that the smaller face is output in the 20th rather than the larger one. Missing a fraction of the face or including small background part in the face may often be insignificant when the face region rather than the segmented image itself is of interest. The larger face in the 20th was output when using the second pattern in Fig. 5(b). It took about 100 seconds with a $1.2 \mathrm{GHz}$ PentiumIII PC for the case using three sets of the weights.

The 100 faces have the statistics: $H$ varies from $-20(340)$ to 61 with mean value 19.6 in the scale of $360, S$ varies from 0.11 to 0.79 with mean value 0.32 , and $V$ varies from 0.28 to 0.93 with mean value 0.64 . Taking advantage of this diversity we can retrieve the frame from a specific binary face pattern and its representative color in a quick way. In the modified system, we allowed $10 \%$ color deviations from the color components of the input face and used 25 or $10 \%$ as the standard deviation of the Gauss function for each RGB color in Eq. (8).

As one of typical cases, when the face in the first frame in Fig. 4 with mean values of $(H, S, V)=(15.7,0.24,0.67)$ was input to the system, we obtained the result in four seconds that the segmented face in the first frame had the highest correlation value 0.80 while it is less than 0.43 for others. As a special case, when the face in the 12 th frame with mean values $(38.7,0.79,0.58)$ was input, we obtained the result in one second that the segmented face in the frame had 0.73 as the correlation value and others had null values. In this case both the hue and the saturation means deviate from the average ones to a great degree, which led to a faster detection of the frame.

\section{Conclusions}

An improved segmentation method using HSV color was presented for face detection in video footages. Based on it, we constructed a face detection system that can detect more than 95 faces out of 100 successfully. We demonstrated its application to image retrieval from video data.

\section{References}

[1] Yang, M-H., Kriegman, D.J., Ahuja, N.: Detecting Faces in Images: A Survey. IEEE Trans. PAMI 24 (2002) 34-58

[2] Rowley, H.A., Baluja, S., Kanade, T.: Neural Network-Based Face Detection. IEEE Trans. PAMI 20 (1998) 23-38

[3] Feraud, R., Bernier, O.J., Viallet, J.-E., Collobert, M.: A Fast and Accurate Face Detector Based on Neural Networks. IEEE Trans. PAMI 23 (2001) 42-53

[4] Schneiderman, H. Kanade, T.: Probabilistic Modeling of Local Appearance and Spatial Relationships for Object Recognition. Proc. CVPR (1998) 45-51 
[5] Osuna, E., Freund, R., Girosi, G.: Training Support Vector Machines: An Application to Face Detection. Proc. CVPR (1997) 130-136

[6] Turk, M.A., Pentland, A.P.: Eigenfaces for Pattern Recognition. J. Cognitive Neuroscience 3 (1991) 71-96

[7] Fröba, B., Ernst, A., Küblbeck, C.: Real-Time Face Detection. Proc. 4th IASTED International Conf. Signal and Image Processing (2002) 497-502

[8] Satoh, S., Nakamura, Y., Kanade, T.: Name-It: Naming and Detecting Faces in News Videos. IEEE Multimedia 6 (1999) 22-35

[9] Wang, D.: Unsupervised Video Segmentation Based on Watersheds and Temporal Tracking. IEEE Trans. Circuits and Systems for Video Technology 8 (1998) 539-545

[10] Toklu, C., Tekalp A.M., Tanjuerdem, A.: Simultaneous Alpha Map Generation and 2D Mesh Tracking for Multimedia Applications. Proc. ICIP 1 (1997) 113-116

[11] Long, F., Feng, D., Peng, H., Siu, W.: Extracting Semantic Video Objects. IEEE Computer Graphics and Applications 21 (2001) 48-55

(a)

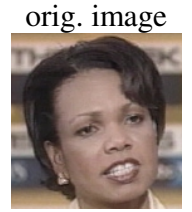

(b)

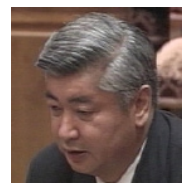

(c)

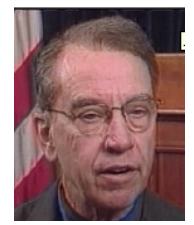

(d)

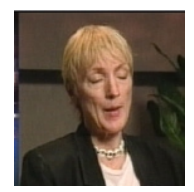

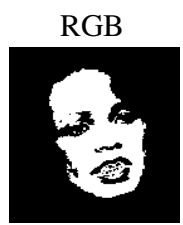

$(1,1,1), 15 \%$

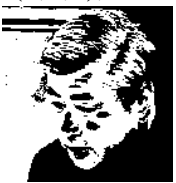

$(2,1,1), 10 \%$

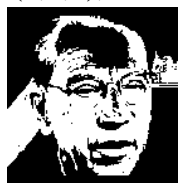

$(2,1,1), 14 \%$

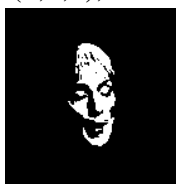

$(1,1,1), 13 \%$

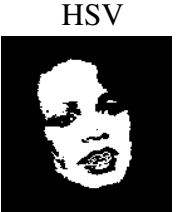

(2,1,2), $7 \%$

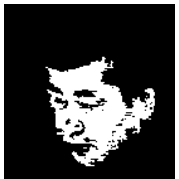

$(5,1,1), 4 \%$

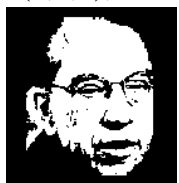

$(2,1,1), 8 \%$

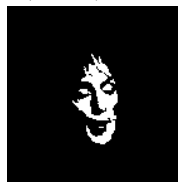

$(10,3,3), 5 \%$

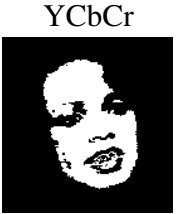

$(1,2,2), 4 \%$

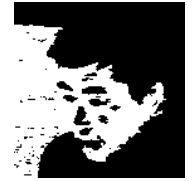

$(1,5,5), 2 \%$

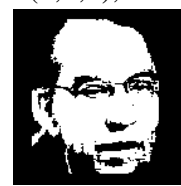

$(1,5,5), 4 \%$

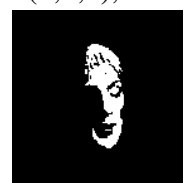

$(2,3,3), 5 \%$

Fig. 3 Examples of segmented images for RGB, HSV, and $\mathrm{YCbCr}$, where three values in the parenthesis are weights on the color components and the fourth value is the segmentation error. 


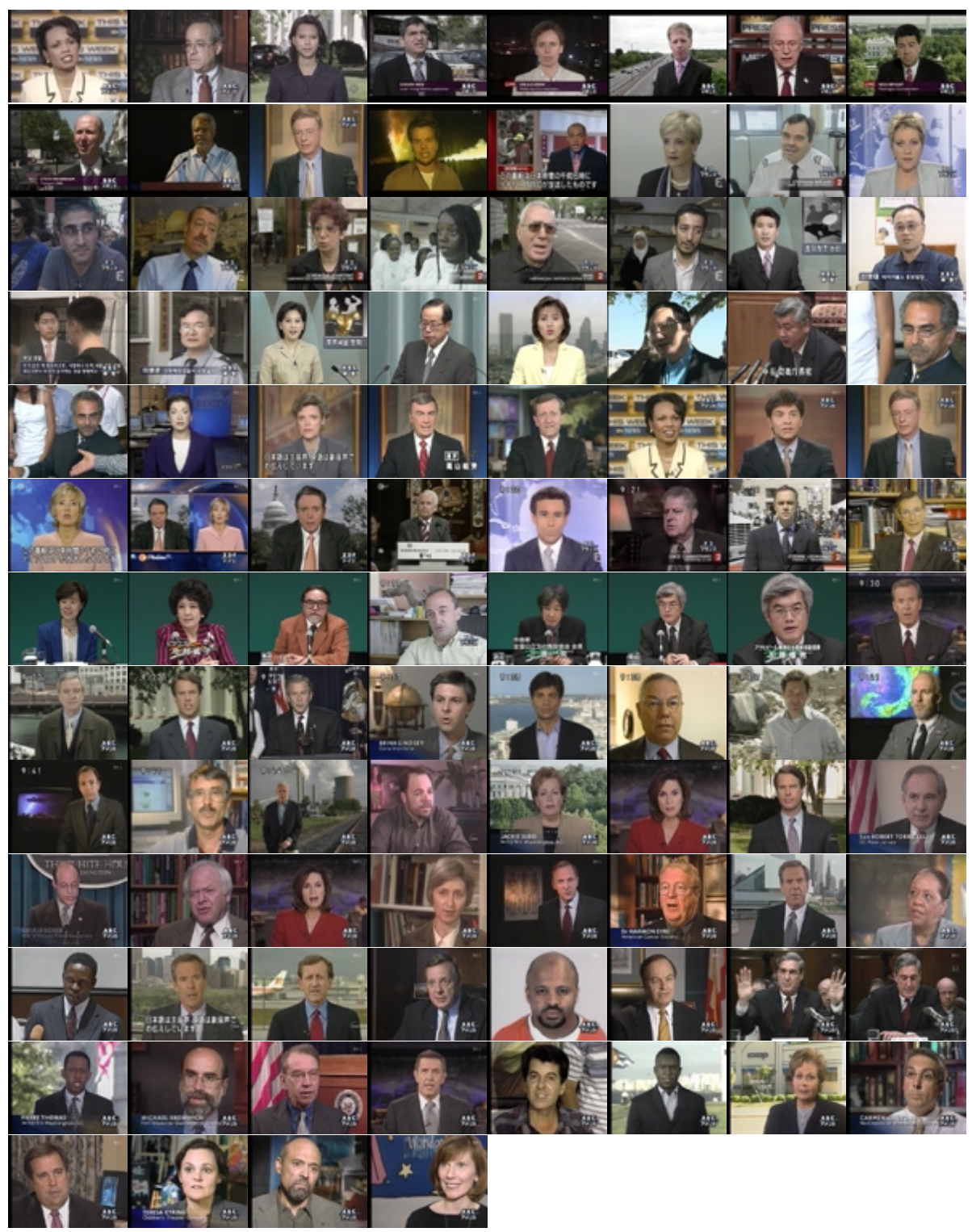

Fig. 4100 frames of the video footage used for our face detection experiments, which was created by editing video footages from various news programs. 


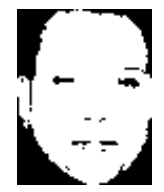

(a)

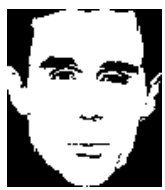

(b)

Fig. 5 Two patterns used as typical face.

\begin{tabular}{|c|c|c|c|c|c|c|c|}
\hline 5 & sis & 8 & 8 & 9 & 6 & 8 & 7 \\
\hline 9 & : & * & 8 & 츱 & 8 & 8 & 9 \\
\hline$\Rightarrow$ & 48 & $B H$ & 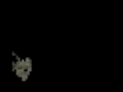 & 합 & 6 & 裙 & ह \\
\hline 9 & 8 & $\theta$ & 5 & 8 & 4 & 5 & 8 \\
\hline 항 & 합 & $\theta$ & 함 & 8 & 6 & $g$ & 9 \\
\hline 8 & $\%$ & 8 & 18 & $\theta$ & 敬 & (6) & 5 \\
\hline 8 & t & 28 & 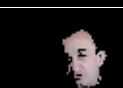 & $\bullet$ & 합 & 8 & $y$ \\
\hline 6 & 하요 & 要 & fes, & 0 & 9 & $\nabla$ & 8 \\
\hline$\theta$ & 8 & $\theta$ & 8 & 8 & 8 & 8 & 6 \\
\hline 9 & $\theta$ & 우 & 9 & $\theta$ & 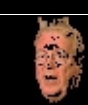 & 9 & 8 \\
\hline s. & 8 & 7 & 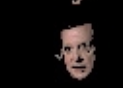 & B & 6 & 할 & 웅 \\
\hline 8 & 8 & बํㅛㅂ & $\theta$ & 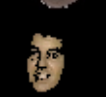 & 6 & 2 & 8 \\
\hline 3 & 8 & 8 & 4 & & & & \\
\hline
\end{tabular}

Fig. 6 Segmention results that include successfully segmented 97 face images and three failed ones on the 18th, 19th and 44th frames. 